\title{
Introduction to Stem Cell Special Section
}

The prospect of utilizing stem cells for clinical applications has generated an enormous amount of enthusiasm in the stem cell research community and has led to a wealth of new data that offer the possibility for practical applications into clinical translation. Recent advances in stem cell imaging has contributed greatly to the ultimate goal to identify and culture specific cell types for regeneration of tissue that had been affected by disease such as Parkinson's, Alzheimer's, heart disease, muscle diseases, and others.

The present issue features original and review articles written by experts in their specific fields who have been invited for their significant contributions to stem cell biology and microscopy. The articles have been selected to represent different aspects of the field and cover novel imaging with multiphoton flow cytometry for the identification of biomarkers and applications to stem cells; neural stem cells in neurospheres, embryoid bodies, and the central nervous system; totipotent ovarian stem cells capable of differentiation into eggs and neuronal cells; hematopoietic stem cell engraftment of the intestinal tumor microenvironment; pluripotent stem cells and reprogrammed cells in farm animals; the hair follicle bulge as niche for adult stem cells; and the role of centrosomes in cell division and differentiation.

It has been a great pleasure to edit this special issue, and I would like to sincerely thank all contributors for their excellent articles and for sharing their unique expertise with the microscopy community. I hope that this special issue will stimulate further advances in stem cell imaging and contribute new data for biomedical applications and regenerative medicine.

Heide Schatten

Department of Veterinary Pathobiology University of Missouri-Columbia 